\title{
A network of topographic numerosity maps in human association cortex
}

\author{
Ben M. Harvey ${ }^{1,2 \star}$ and Serge O. Dumoulin ${ }^{1,3}$
}

\begin{abstract}
Sensory and motor cortices each contain multiple topographic maps with the structure of sensory organs (such as the retina or cochlea) mapped onto the cortical surface. These sensory maps are hierarchically organized. For example, visual field maps contain neurons that represent increasingly large parts of visual space with increasingly complex responses'. Some visual neurons respond to stimuli with a particular numerosity the number of objects in a set. We recently discovered a parietal topographic numerosity map in which neural numerosity preferences progress gradually across the cortical surface ${ }^{2}$, analogous to sensory maps. Following this analogy, we hypothesized that there may be multiple numerosity maps. Numerosity perception is implicated in many cognitive functions, including foraging ${ }^{3}$, multiple object tracking ${ }^{4}$, dividing attention, decision-making ${ }^{6}$ and mathematics ${ }^{7-9}$. Here we use ultra-highfield (7 Tesla, 7T) functional magnetic resonance imaging (fMRI) and neural-model-based analyses to reveal numerosityselective neural populations organized into six widely separated topographic maps in each hemisphere. Although we describe subtle differences between these maps, their properties are very similar, unlike in sensory map hierarchies. These maps are found in areas implicated in object recognition, motion perception, attention control, decision-making and mathematics. Multiple numerosity maps may allow interactions with these cognitive systems, suggesting a broad role for quantity processing in supporting many perceptual and cognitive functions.
\end{abstract}

Topographic maps have an orderly organization of neurons with similar functions. The close proximity of neurons with similar functions minimizes local connection lengths to increase neural processing efficiency ${ }^{10-12}$. Furthermore, topographic maps allow simple one-to-one projections between maps. Finally, most neural processes are context-dependent. Topographic maps allow easy computations of context through comparisons between neighbouring neurons. Therefore, topographic organization has several benefits and gives a theoretical framework to explain why maps emerge in cognitive processing, as we recently demonstrated ${ }^{2}$ and extend here. Together with this numerosity map, we also demonstrated that another quantity, object size, is processed in a distinct object size map that largely overlaps with this parietal numerosity map, showing correlated numerosity and object size preferences ${ }^{13}$.

The parietal numerosity map that we have described encompasses part of a network implicated in numerosity processing, extending into occipital, parietal and frontal areas ${ }^{14-19}$. The fine-scale organization elsewhere in this numerosity network is unknown. We hypothesize that, like sensory maps, a hierarchy of several numerosity maps throughout human association cortices underlies this numerosity network. To investigate this hypothesis, we adapted our approach to reconstruct numerosity maps throughout the brain.

We displayed visual stimuli of changing numerosity while collecting ultra-high-field (7T) fMRI data covering the occipital, parietal, posterior-superior frontal and temporal lobes. We distinguished between responses to numerosity and co-varying stimulus features using several stimulus configurations ${ }^{2,16}$. We summarized the fMRI responses using numerosity-selective population receptive field (pRF) models with two parameters: preferred numerosity and tuning width. We consistently found six numerosity maps for which these models explain responses very well (mean variance explained $=66 \%$, corresponding to $P=0.0022$; see Methods) (Fig. 1a; Supplementary Fig. 1). These numerosity maps were often widely separated but showed very similar patterns of response. Each numerosity map contained very different responses separated by short distances (1-2 cm) (Fig. 1). Logarithmic Gaussian tuning functions explained slightly more response variance than linear Gaussian functions in all maps (Wilcoxon signed-rank test: NTO $P<10^{-20}, z=10.0, \Delta=1 \%, n=1559$; NPO $P=10^{-7}, z=5.2$, $\Delta=0.1 \%, n=647 ;$ NPC1 $P<10^{-20}, z=29.0, \Delta=1 \%, n=1675$; NPC2 $P<10^{-20}, z=34.0, \Delta=2 \%, n=1186$; NPC3 $P<10^{-20}, z=16.6$, $\Delta=1 \%, n=885$; NF $P<10^{-20}, z=28.7, \Delta=1 \%, n=1187$, false discovery rate (FDR)-corrected for multiple comparisons; degrees of freedom $(\mathrm{DF})=n-1$; see below for abbreviations), consistent with previous reports from some maps ${ }^{2,6,13}$. As we recently reported for NPC1 (B.M.H. and S.O.D., manuscript in preparation) responses from all maps were better captured by numerosity-selective responses than by selective responses to other stimulus features that co-vary with numerosity in some stimulus configurations, or by visual field stimulation (Wilcoxon signed-rank test of variance explained, all $P<10^{-6}$, all $z>4.8$, all $\Delta>7 \%$, all $n>38$, all $\mathrm{DF}>37$ ) (Supplementary Fig. 2).

Projecting each recording site's preferred numerosity onto the inflated cortical surface revealed six orderly topographic numerosity maps in each hemisphere (Fig. 2a, Supplementary Fig. 3). In each map, numerosity preferences changed gradually across the cortical surface, repeatably across subjects, scanning sessions and stimulus configurations (Supplementary Figs 4 and 5). We named these numerosity maps after their anatomical locations, following naming conventions for extrastriate visual field maps ${ }^{20}$. We preceded their locations with ' $\mathrm{N}$ ' for numerosity. Moving from posterior to anterior, the first numerosity map (NTO for 'numerosity temporooccipital') lay at the lateral temporo-occipital junction, between the inferior temporal and lateral occipital sulci, posterior-superior to the preoccipital notch. NTO's centre was at Montreal Neurological Institute (MNI) $x, y, z$ coordinates $44(7),-75(1),-4(3)$ in the right

${ }^{1}$ Experimental Psychology, Helmholtz Institute, Utrecht University, Heidelberglaan 1, 3584 CS, Utrecht, The Netherlands. ${ }^{2}$ Faculty of Psychology and Education Sciences, University of Coimbra, Rua do Colégio Novo, 3001-802, Coimbra, Portugal. ${ }^{3}$ Spinoza Centre for Neuroimaging, Meibergdreef 75, 11005 BK, Amsterdam, The Netherlands. *e-mail: b.m.harvey@uu.nl 
a

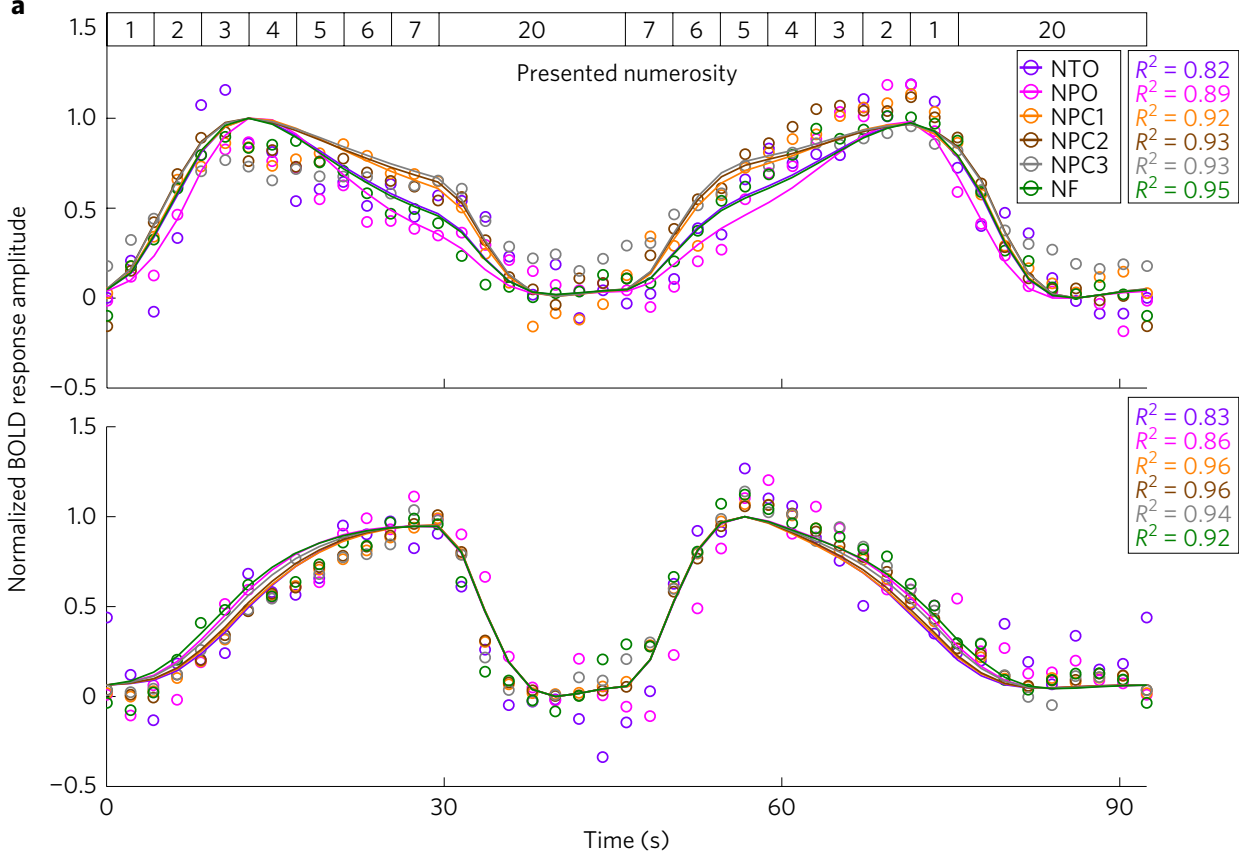

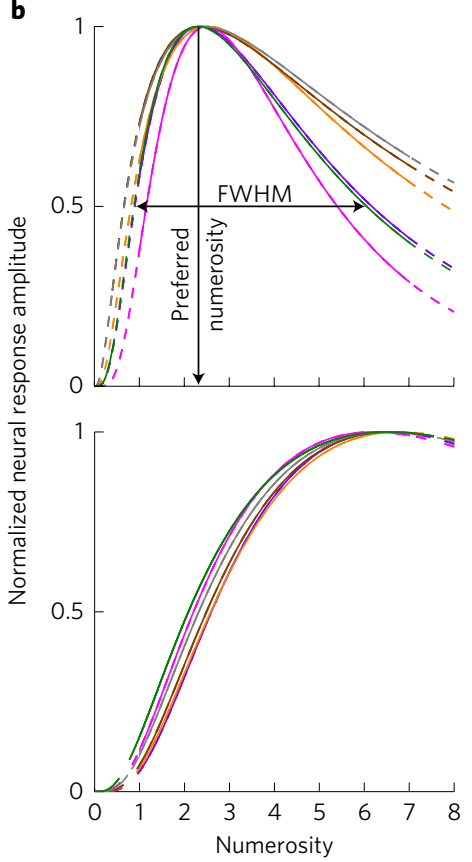

Figure 1 | Similar responses to numerosity in several brain regions. a, Varying stimulus numerosity (top bar) elicited different responses within each numerosity map (different colours). In the upper panel, the largest response amplitude occurred after presentation of low numerosities, whereas in the lower panel the largest responses followed higher numerosities, considering the haemodynamic response delay. We captured these different responses using numerosity-selective neural models, which captured high proportions of the variance $\left(R^{2}\right)$, in all time courses $\left(R^{2}>0.8\right)$. Between numerosity maps, responses and corresponding neural models were similar. Points represent response amplitudes averaged over all stimulus

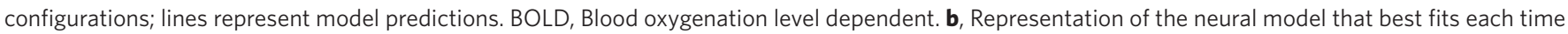
course. The model described a logarithmic Gaussian tuning function with two parameters: preferred numerosity and tuning width defined by the full-width at half-maximum (FWHM). Different model parameters explained the differences seen in the two panels in a, capturing a similar proportion of response variance. Dashed lines are predicted neural responses beyond the tested stimulus range.

hemisphere and $-42(3),-77(3),-3(8)$ in the left hemisphere (values are given as mean (SD)). The second numerosity map (NPO) lay at the superior end of the parieto-occipital sulcus (right 25(5), $-82(4), 34(6)$, left $-23(4),-80(5), 32(7))$. The third, fourth and fifth numerosity maps (NPC1, NPC2 and NPC3) lay in and around the postcentral sulcus. NPC1 lay on the gyrus posterior to the superior postcentral sulcus (right 22(5), -61(7), 60(5), left -22(4), $-59(11), 61(8))$. NPC1's location and orientation were very similar to our previous reports in the same subjects ${ }^{2,13}$. NPC2 and NPC3 lay in the postcentral sulcus, superior and inferior (respectively) to its junction with the intraparietal sulcus (right 33(3), -40(4), 52(7), left $-38(3),-43(8), 48(8)$ and right 45(10), -30(6), 40(4), left $-48(6)$, $-29(5), 34(6))$. The sixth numerosity map (NF) lay at the junction of the precentral and superior frontal sulci (right 24(3), -11(5), $52(6)$, left $-22(3),-11(6), 50(8))$. We found further numerosityselective areas in some cases, but not consistently between scanning sessions or subjects.

To quantify each numerosity map's organization, we sorted each map's recording sites by their distance to the map borders with the lowest and highest preferred numerosities present (the white lines in Fig. 2a and Supplementary Fig. 3). We then plotted preferred numerosity against cortical distance for each stimulus configuration and their average (Fig. 2b, Supplementary Figs 4 and 5). In each numerosity map, preferred numerosity changed systematically and repeatably across the cortical surface in each stimulus configuration and scanning session, although less consistently in left-hemisphere maps (Supplementary Fig. 5). We recorded each of six numerosity maps in four stimulus configurations in five subjects $(n=120$ measures per hemisphere). Permutation analysis revealed significant progressions of preferred numerosity with cortical distance in 107/120 right-hemisphere measures and 98/120 left-hemisphere measures (FDR-corrected). A three-way ANOVA ( $n=240$ numerosity map measures) revealed differences in the slope of the cortical numerosity progression between maps, stimulus configurations and hemispheres (map effect $P=0.0001, F=5.7, D F=5$; stimulus configuration effect $P=4 \times 10^{-7}, F=11.6, \mathrm{DF}=3$; hemisphere effect $P=0.015, F=6.0, \mathrm{DF}=1)$. Subsequent multiple comparison test $^{21,22}$ revealed that NF had less preferred numerosity progression than other maps, the constant-perimeter stimulus configuration produced less preferred numerosity progression than other configurations, and left-hemisphere maps had less preferred numerosity progression than right. Similarly, significant numerosity progressions were less frequent in NPO and NF than in other numerosity maps, in the constant-perimeter and high-density stimulus configurations, and in the left hemisphere (Supplementary Table 1).

Preferred numerosities within each numerosity map were well correlated between stimulus configurations recorded on different days (Supplementary Fig. 6), reflecting common topographical organization across stimulus configurations and repeated measures. However, numerosity preferences from the configuration with constant-perimeter stimuli were consistently less well correlated with preferences from other stimulus configurations $s^{2,13}$.

The numerosity maps for the left and right hemispheres represented different numerosity ranges. Except in NTO, left-hemisphere maps contained more recording sites with low preferred numerosities (below about three) than right-hemisphere maps, while righthemisphere maps contained more high preferred numerosities (Figs $2 \mathrm{~b}$ and $3 \mathrm{a}$ ). We used the upper quartile of preferred numerosities in each map to summarize this difference. A three-way analysis of variance, ANOVA ( $n=60$ numerosity maps), for effects of 
a Left hemisphere Subject 1 (inferiortemporals
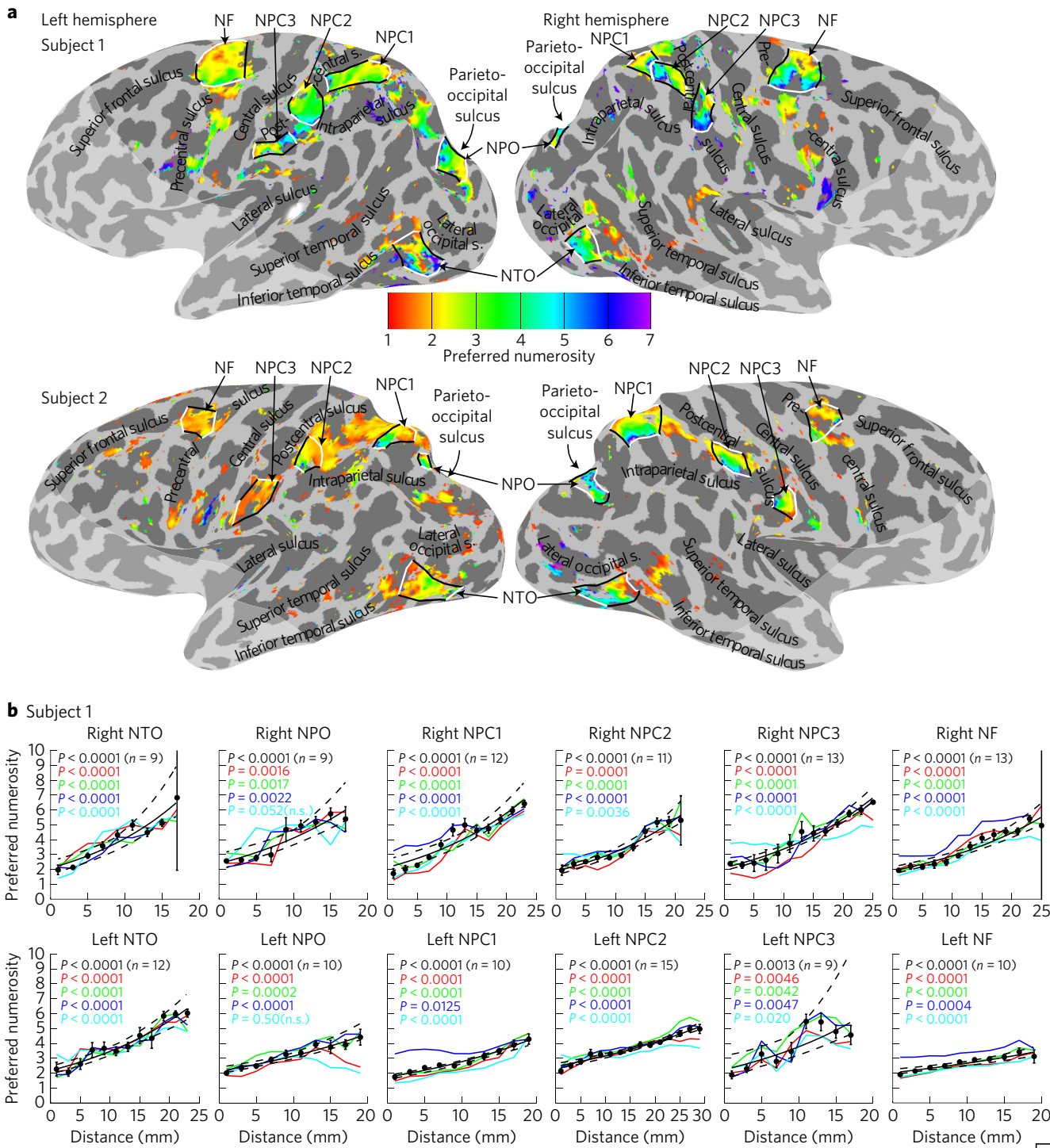

Left NPC1

Left NPC2
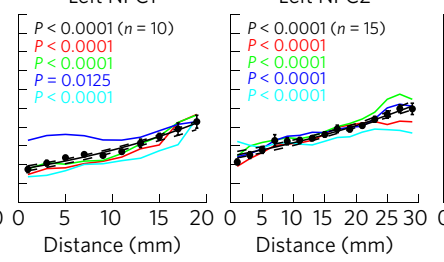

Left NPC3

Left NF
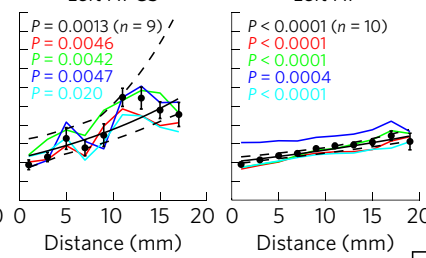

Subject 2
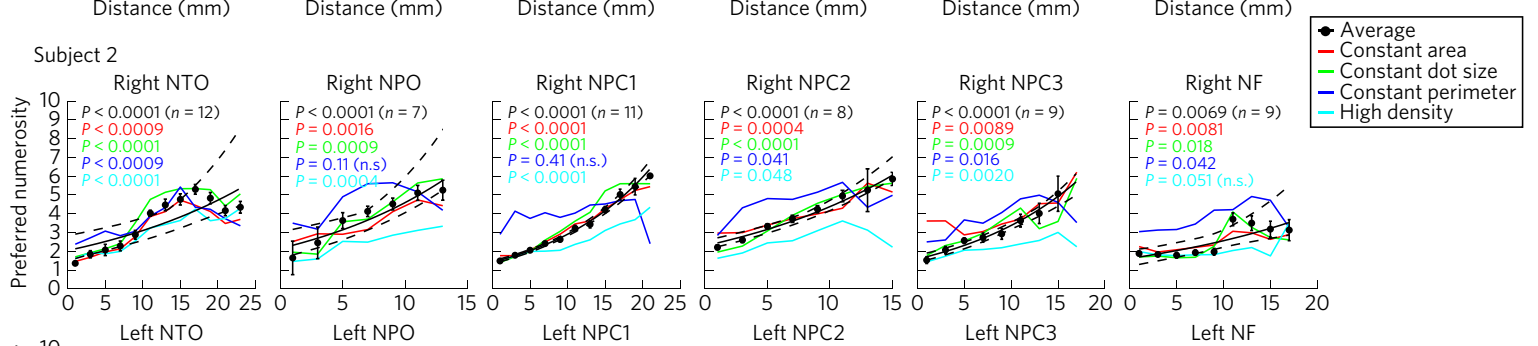

$\begin{aligned} P & =0.0069(n=9) \\ P & =0.0081\end{aligned}$

$\begin{aligned} P & =0.0069(n) \\ P & =0.008 \\ -P & =0.018 \\ P & =0.042\end{aligned}$
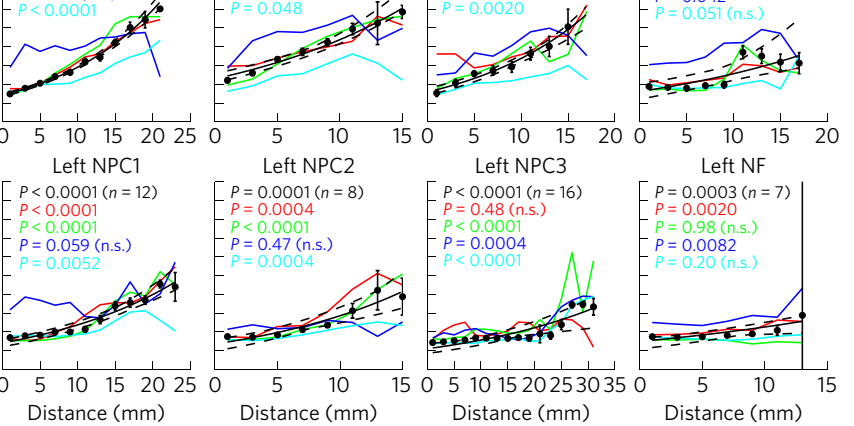

Figure 2 | Numerosity map network. a, Preferred numerosity in sites of significant numerosity-selective responses (variance explained $>30 \%, P<0.0371$; FDR-corrected for multiple comparisons). In each hemisphere, there were several topographic numerosity maps, that is, extended areas where preferred numerosity changed gradually across the cortical surface. Colours show each recording site's preferred numerosity. White lines connect recording sites with the highest or lowest preferred numerosity present in each numerosity map. Black lines show borders of numerosity maps. Text labels show major sulci. The lighter shaded region of each hemisphere was outside the fMRI recording volume. b. Preferred numerosity of recording sites organized into bins by distance along each numerosity map's cortical surface between the white lines in a. In each numerosity map, preferred numerosity changed systematically and repeatably in each stimulus configuration. We fitted the mean preferred numerosities (black circles) for the distance bins with a logarithmic function (black line) with 95\% confidence intervals to the fit (dashed black lines) determined by bootstrapping. Error bars show the standard error of the mean for each bin. Different stimulus configurations are represented as coloured lines joining the configuration-specific bin means. Coloured text gives probability of the observed change from permutation analysis, FDR-corrected. n.s., not significant. 

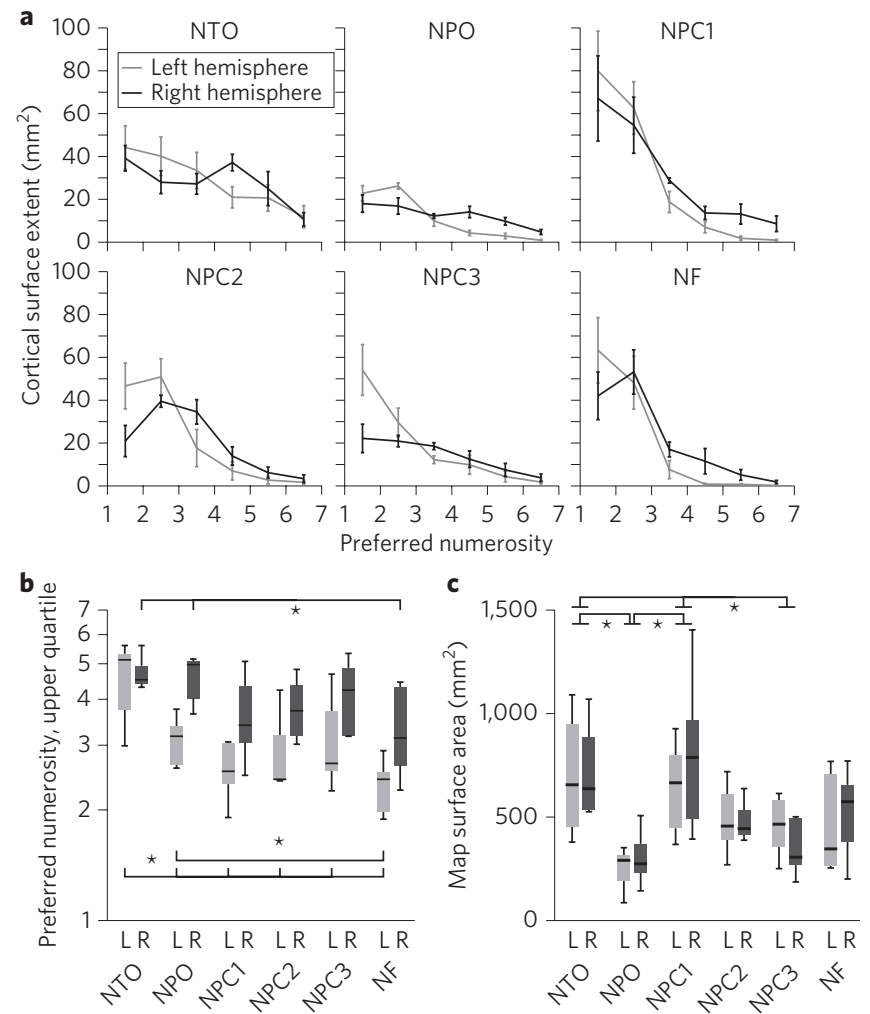

Figure 3 | Differences in numerosity range and surface area between numerosity maps and hemispheres. a, A larger proportion of each left-hemisphere numerosity map had low preferred numerosities (below about three), while a larger proportion of each right-hemisphere map had higher preferred numerosities. Error bars show the standard error of the mean. b, Except in NTO, right-hemisphere numerosity maps (dark grey) represented a higher range of numerosities than their paired left-hemisphere maps (light grey), compared using the upper quartiles of the preferred numerosities present. There were also differences in the preferred numerosity distribution between numerosity maps and subjects within each hemisphere (see text). Brackets and stars show significant differences in subsequent multiple comparisons between maps in the same hemisphere: all brackets to the left of the star are significantly different from all brackets to the right of the star $(P<0.05)$. c, Numerosity map surface areas differed between numerosity maps, but not between hemispheres. NTO and NPC1 were the largest numerosity maps, NPO was smallest, and other maps had similar intermediate surface areas. Brackets and stars show significant differences in subsequent multiple comparisons with maps grouped across hemispheres: all brackets to the left of the star are significantly different from all brackets to the right of the star $(P<0.05)$. Horizontal lines are means, boxes are interquartile ranges of values from different subjects (not interquartile ranges of preferred numerosities), error bars are most extreme values.

hemisphere, map and subject first revealed that upper quartiles differ between hemispheres $\left(P=2 \times 10^{-9}, F=54.0, \mathrm{DF}=1\right)$. Subsequent multiple comparison tests revealed that right-hemisphere upper quartiles were significantly higher than those for the left hemisphere, except in NTO (Fig. 3b). Thus right-hemisphere numerosity maps typically had higher and broader distributions of preferred numerosity, although NTO had similar distributions across hemispheres.

The same three-way ANOVA also revealed that upper quartiles of preferred numerosity distributions differed between subjects and between numerosity maps in the same hemisphere (map effect $P=1 \times 10^{-8}, F=14.4, \mathrm{DF}=5$; subject effect $P=3 \times 10^{-8}, F=15.6$, $\mathrm{DF}=4$ ) (Fig. 3b). Subsequent multiple comparison tests showed that left NTO had higher preferred numerosity distributions than other left-hemisphere maps, which had similar distributions.
More posterior right-hemisphere maps (NTO and NPO) had significantly higher preferred numerosity distributions than anterior maps (NF). Right parietal maps (NPC1, NPC2 and NPC3) had intermediate preferred numerosity distributions that did not differ significantly from either posterior or anterior maps. So high numerosity preferences were primarily found in right posterior numerosity maps.

Surface areas of numerosity maps also differed (Fig. 3c). We found no hemisphere difference or interactions, so used a two-way ANOVA across both hemispheres for effects of map and subject ( $n=60$ numerosity maps). This revealed differences in map surface areas between maps and between subjects (map effect $P=1.7 \times 10^{-6}$, $F=9.7, \mathrm{DF}=5$; subject effect $P=0.0008, F=5.6, \mathrm{DF}=4)$ with no interaction. Subsequent multiple comparison tests revealed that NTO and NPC1 were the largest numerosity maps, NPO and NPC3 were significantly smaller, and NPC2 and NF had intermediate surface areas. Therefore differences between map surface areas did not suggest a progression from posterior to anterior.

In responses averaged across stimulus configurations, numerosity tuning widths consistently increased with preferred numerosity in each numerosity map (Fig. 4a, Supplementary Fig. 7). This increase was significant in 23/30 right-hemisphere numerosity maps and 16/30 left-hemisphere maps (FDR-corrected).

Maps in the sensory cortex, and in particular the visual cortex, show large systematic differences in tuning widths (that is, receptive field sizes). Do numerosity tuning widths likewise differ between numerosity maps? To examine average tuning widths without biases arising from different preferred numerosity distributions, we quantified the fitted progression of tuning widths at a preferred numerosity of three ${ }^{23}$. We found no hemisphere difference or interactions, so used a two-way ANOVA across both hemispheres for effects of map and subject ( $n=60$ numerosity maps). This revealed effects of map and subject on tuning widths (map effect $P=0.0045, F=3.9, \mathrm{DF}=5$; subject effect $P=0.0024, F=4.8, \mathrm{DF}=4$ ) with no interaction (Fig. $4 \mathrm{~b}$ and c). Subsequent multiple comparison tests within each hemisphere reveal that NTO and NPO had the largest tuning widths, NF had significantly smaller tuning widths, and other maps had intermediate tuning widths that did not differ significantly from NTO, NPO or NF. So there may be a slight tuning width decrease from posterior to anterior maps, but this is not as clear as the decrease in preferred numerosity distribution.

Finally, we asked how numerosity map locations related to visual field map locations (Supplementary Figs 8 and 9). All numerosity maps lay in or near visually responsive brain areas and partially overlapped one or more extrastriate visual field maps. However, numerosity maps were not limited to the representation of the central visual field, where numerosity stimuli were presented. Furthermore, no numerosity map shared its borders with a visual field map. Finally, relative locations of each numerosity map and its nearby visual field maps differed considerably between subjects and hemispheres. We are therefore confident that our numerosity maps do not reflect the organization of visual field maps.

NTO overlaps with parts of visual field maps LO2, TO1 and/ or TO2. Posterior-superior NTO typically fell beyond the parts of LO2 and TO1 covered by our visual field mapping stimulus. NPO overlapped with parts of IPS0 and/or IPS1. NPC1 overlapped with parts of IPS2, IPS3 and/or IPS4. NPC2 overlapped with parts of IPS4 and IPS5, often extending beyond IPS5 and beyond the eccentricity range that we stimulated. NPC3 lay anterior and inferior to IPS5, beyond any previously described visual field map, but partially overlapping with selective responses to visual field position that suggest further undocumented inferior postcentral visual field map(s). Anterior NF typically partially overlapped with hFEF (the putative human frontal eye field map ${ }^{24}$ ).

This numerosity map network's extent and overlap with other brain areas indicate that human number and quantity processing 
a $\quad$ Subject 1
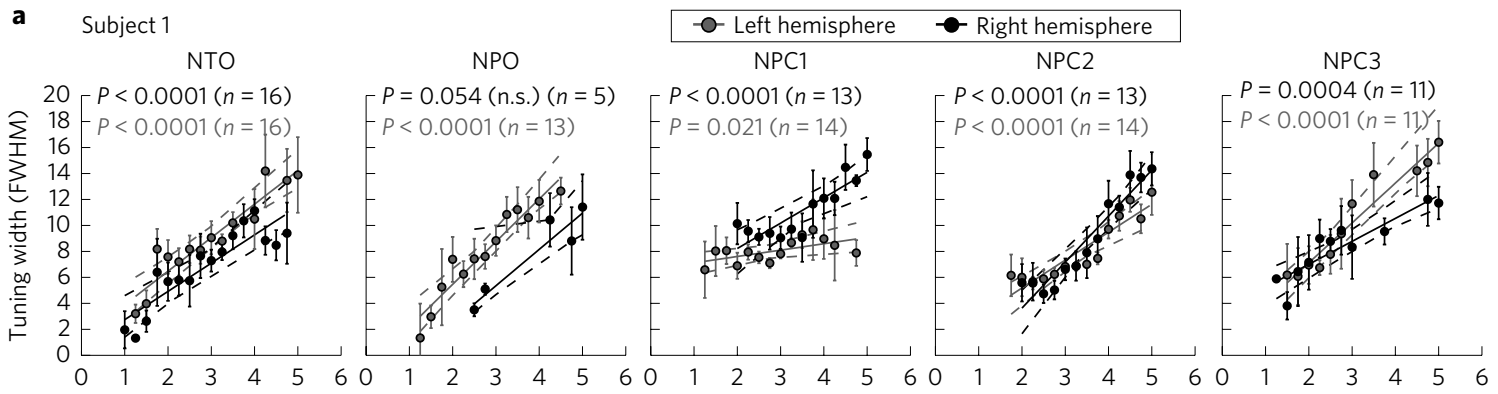

NPC3

$\mathrm{NF}$
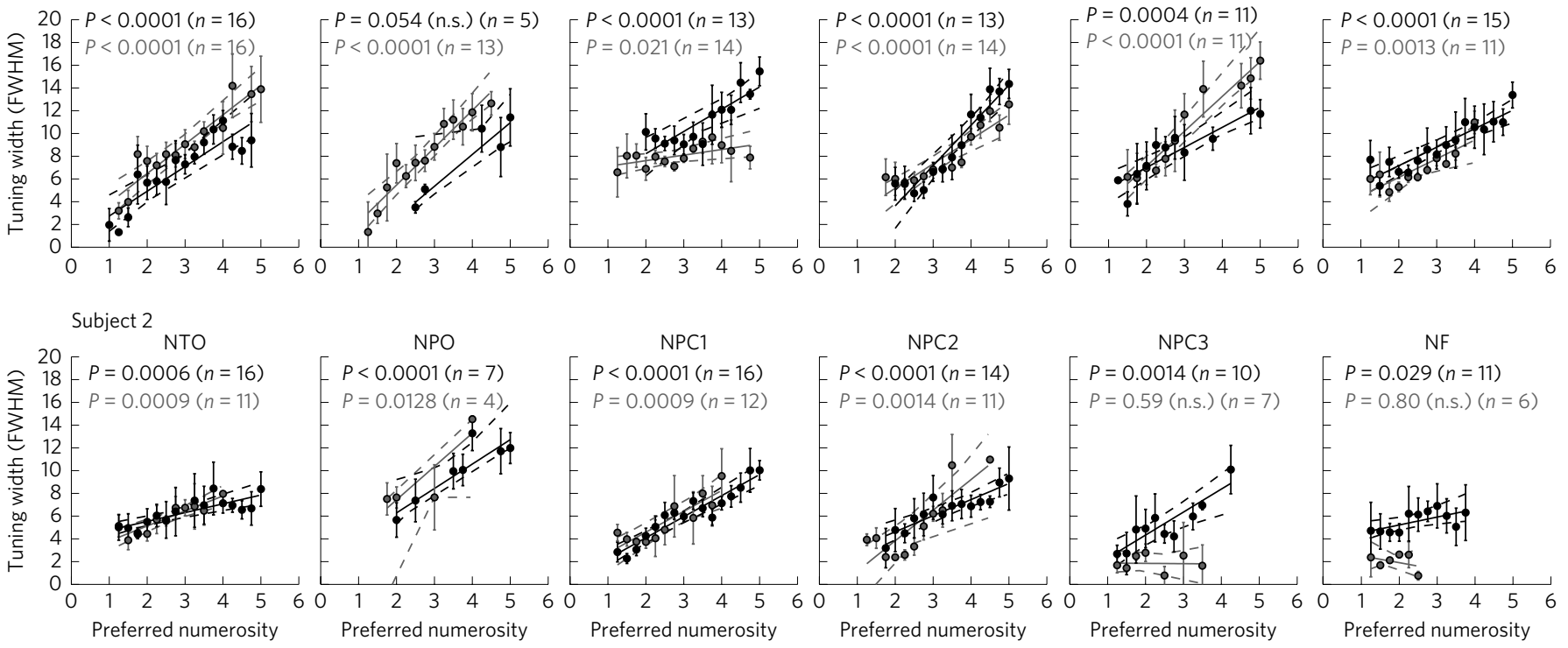
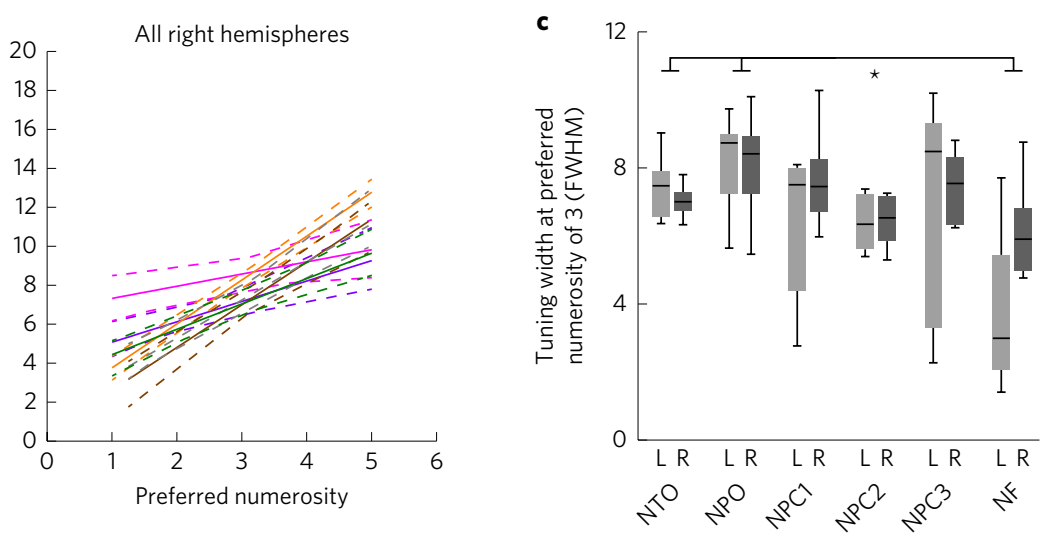

Figure 4 | Numerosity tuning widths. a, Progression of population tuning width with preferred numerosity in each numerosity map shown in Fig. 2. We fitted mean tuning widths (circles) for preferred numerosity bins with a linear function (solid lines) with $95 \%$ confidence intervals to the fit (dashed lines) determined by bootstrapping. Error bars are standard errors. The text gives the probability of the observed change from permutation analysis, FDR-corrected. b, Tuning widths grouped across subjects in each hemisphere. c, Tuning widths differ slightly between maps in both hemispheres (see main text). Brackets and stars show significant differences in subsequent multiple comparisons with maps grouped across hemispheres: all brackets to the left of the star are significantly different from all brackets to the right of the star $(P<0.05)$.

may interact with several perceptual and cognitive functions. While processing in common areas implies interactions between neural systems ${ }^{13,25,26}$, different systems may be located together without interacting.

As our stimuli were presented visually, it is not surprising that responses were in visual areas. There are strong links between perception of numerosity and visual space or motor response loca$\operatorname{tion}^{27,28}$, probably mediated by working-memory organization ${ }^{29,30}$. So overlapping responses to numerosity (or its working-memory footprint) and visual or motor location may underlie the cognitive spatial number line $e^{2}$. However, we find no clear relationship between numerosity and visual space preferences. Indeed, we find more high preferred numerosities in the right hemisphere, which primarily represents left visual and motor space, contrary to the usual association between high numbers and right visual or motor space $^{31}$. We therefore find no obvious mechanism linking visual or motor space representations with numerosity representations.

The numerosity map network overlaps considerably with the fronto-parietal attention network of the intraparietal, postcentral and precentral sulci. Numerosity perception is more difficult with higher numerosities ${ }^{6,18}$. Could tuned numerosity-selective responses reflect tuned responses to attentional load? This seems unlikely. First, our subjects performed no task with the displayed numerosities, and numerosity changed predictably. Second, attentional load should not differ in the constant-perimeter stimulus configuration, but preferred numerosities differ here. Finally, tuned responses to attentional load have never been reported. Therefore responses to numerosity do not straightforwardly reflect responses to attentional load.

Nevertheless, links between numerosity and attention seem likely. Display numerosity affects the attentional capacity for object individuation, suggesting that numerosity representations guide attention's spread between multiple objects ${ }^{5}$. Visual attention may use numerosity preferences to individuate objects and distribute attentional foci between them. Finally, stimuli attract attention, so numerosity-selective responses may reflect stimulus properties, or the distribution of attention that the stimulus generates. Likewise, responses that are selective for visual field position may reflect the position of the stimulus and/or the attention the stimulus attracts. Visual processing and attention affect each other, 
and attention may be an inherent component of stimulus-driven responses in higher visual processing.

Few recording sites had numerosity preferences above five. These numerosity preferences extend little beyond the subitizing range. While this low numerosity range is widely used to investigate numerosity-selective responses in animals ${ }^{6,16,17}$, most human fMRI and behavioural studies use higher numerosities. The 'approximate number system' for higher numerosities depends less on attention ${ }^{32}$ and may not rely on the system we characterize here ${ }^{4,33,34}$. The ability to decode numerosities beyond seven ${ }^{14,15}$ suggests that responses to higher numerosities have spatial structure at fMRI resolutions. Alternatively, decoding of higher numerosities may depend on differential activation of sites with lower preferred numerosities.

NTO's location in and near MT+ (visual field maps TO1 and TO2) and lateral occipital areas (LO1 and LO2) implicates numerosity in motion processing and object processing, respectively. Interactions between numerosity-selective and motion direction/speed-selective populations may aid object individuation in multiple object tracking ${ }^{4}$. Likewise, the number of features within an object may help us to perceive a face, or to distinguish between rectangles and triangles.

Quantity processing may also guide decision-making ${ }^{6}$ and support mathematical cognition ${ }^{7-9}$. The areas involved in these advanced and complex cognitive functions are less specifically localized, and seem to involve extensive networks that support several related functions. We do not map these, so cannot draw close links to areas supporting decision-making and mathematical cognition. Nevertheless, the intraparietal sulcus (IPS) is often implicated in mathematical calculation ${ }^{35,36}$ and both prefrontal and intraparietal areas in decision-making ${ }^{37,38}$.

Right-hemisphere numerosity maps represent higher and broader numerosity ranges than left-hemisphere maps, except NTO. This hemispheric difference supports previous reports of numerosity processing lateralization ${ }^{2,13,39}$, and extends this principle beyond NPC1 and the IPS. The proportion of high numerosity preferences present also decreases from posterior to anterior numerosity maps, although the functional significance of this trend is unclear. These differences result in small ranges of numerosity preferences in the left hemisphere's anterior maps, with the upper quartile in left NF dropping as low as two. Therefore, numerosity preference progressions have very small effect sizes in some maps. However, recording sites with low numerosity preferences may contain information about higher numerosities: these sites may contain individual neurons with higher numerosity preferences, and neurons with low numerosity preferences respond to changes between higher numerosities.

Where numerosity preferences ranges and the slope of their cortical progression decrease, the slope and frequency of significant numerosity preference progressions also decrease, with only $60 \%$ of left NF measures showing a significant progression. Significant progressions are also less frequent in NPO, which has fewer recording sites from to quantify progressions. So differences in the slope and frequency of significant numerosity progressions (Supplementary Table 1) are linked to differences in preferred numerosity range and map surface area (Fig. 3).

The slope and frequency of significant numerosity preference progressions are lower in the constant-perimeter stimulus configurations. Numerosity preferences measured with this configuration are also less well correlated with those from other configurations. We have previously shown that NPC1 voxels have object size preferences that are positively correlated with numerosity preferences ${ }^{13}$. The constant-perimeter configuration shows small numerosities with very large object sizes and vice versa. Here, recording sites that prefer small numerosities and small object sizes may respond maximally to a larger numerosity because it has smaller objects. This reduces the numerosity preference progressions measured with stimulus configuration and the correlation to measures from other stimulus configurations. Finding this effect outside NPC1 suggests that other numerosity maps may likewise have objectsize-selective responses.

Anterior visual field maps generally contain neurons with larger tuning widths (receptive field sizes) than posterior visual field maps. Other sensory processing hierarchies show similar progressions. Successive integration of visual information allows detection of more complex features by analysing successively larger areas of visual space. Numerosity tuning widths do not increase similarly, and indeed decrease slightly from posterior to anterior numerosity maps. Successive integration, if any, may therefore sharpen frontal numerosity representations. These are more closely linked to behavioural performance ${ }^{40,41}$, so finer tuning widths here may improve behavioural performance. Alternatively, multiple numerosity maps may instead aid interactions with multiple perceptual and cognitive systems without successive integrations

Sensory and motor topographic maps are typically grouped in specific regions, allowing interactions over minimal distances. However, the numerosity maps are widely separated. This broad distribution may also aid interactions with several perceptual and cognitive systems.

Individual subjects differ in several properties of the numerosity maps. Both map surface areas and the upper quartile of preferred numerosities differ between subjects. It is tempting to speculate that some individual difference in numerosity map properties might lead to some individual difference in numerical abilities. However, we remain sceptical of this link. Several behavioural measures (for example subitizing range, accuracy, reaction time or any measure of mathematical abilities) could be correlated with any numerosity map property (for example numerosity map surface area, range of preferred numerosities, or tuning widths), in any of six maps in either hemisphere. Such analyses would also require far more subjects than we test here.

The positions of all numerosity maps are similar across subjects, as are the orientations of NPO and NPC1. However, the orientations of other maps vary. This variability resembles that of visual field maps. While early visual field maps are fairly consistently oriented, the frontal visual field maps hFEF and DLPFC show variable orientation between hemispheres and individuals ${ }^{24}$. However, both higher extrastriate visual field map and numerosity map orientations in each hemisphere are repeatable across independent scanning sessions, so we are confident of these orientations. We speculate that the increasing variability of anterior topographic map orientations may arise because these maps are not constrained by links to the orientations of neighbouring visual field maps and major neuronal pathways such as the optic radiation.

Some hemispheres contain multiple numerosity maps around a region where other hemispheres contain only one. This is clearest around NPC1, where further areas of numerosity selective responses were common. These may represent development of further numerosity maps in some subjects, particularly considering that the postcentral sulcus contains multiple numerosity maps in all subjects.

Macaque lateral prefrontal cortex (LPFC) and anterior inferior temporal cortex (AITC) ${ }^{17}$ recordings suggest numerosity-selective responses in temporal and frontal areas that our scans do not cover. However, it is difficult to predict where homologous locations lie in macaques and in the greatly expanded human association cortices: macaque LPFC and AITC may be homologues of our NF and NTO maps respectively.

Macaque studies describe numerosity-selective responses in the IPS, rather than the postcentral sulcus where we find three numerosity maps. Macaques lack clearly distinguished postcentral and intraparietal sulci, so numerosity-selective responses in macaque IPS may be homologues of our NPC numerosity maps. fMRI numerosity mapping studies in macaques may clarify questions of homology, as they have in visual field mapping ${ }^{42-44}$. 
Human fMRI studies consistently describe IPS activation during numerosity discriminations tasks ${ }^{14,18}$, while our NPC numerosity maps lie in the postcentral sulcus. It is unclear whether previously described IPS locations correspond to NPC numerosity maps. Recent studies with careful response localization on the cortical surface show numerosity information at NPC map locations ${ }^{15}$, or at NPC map locations together with the IPS ${ }^{14}$. However, IPS activation probably reflects responses to comparison tasks (which our subjects did not perform) rather than numerosity ${ }^{45-47}$. Furthermore, most studies use larger numerosities than the preferred numerosities that we find.

Topographic organization is common to processing of sensory inputs, numerosity and other quantities. Numerosity maps form an extensive representation of quantity information throughout human association cortex. This includes areas involved in several cognitive and perceptual functions: visual motion processing, object recognition, attentional control, decision-making and mathematics. As such, an extensive system for quantity processing may form a major link between human perceptual systems and higher cognition.

\section{Methods}

Subjects. We present data from five human subjects (all male, aged 25-39 years) One was left-handed. All were well educated, with good mathematical abilities. All had normal or corrected to normal visual acuity. All were trained with tasks requiring numerosity judgements before scanning. All gave written informed consent. These subjects were used in a previous study ${ }^{13}$, which included a small subset of this data. We have previously used the same number of subjects to characterize numerosity maps ${ }^{13}$. Our approach provides high statistical confidence in numerosity map locations and characteristics in each individual subject. We used multiple subjects to demonstrate reproducibility and generalization across subjects. We used four stimulus configurations on different days to demonstrate reproducibility over independent data collections. All experimental procedures were cleared by the ethics committee of University Medical Center Utrecht.

Numerosity stimuli. Following protocols described in our previous studies ${ }^{2,13}$, we presented visual stimuli by back-projection on a $15 \times 9 \mathrm{~cm}$ screen inside the MRI bore. Subjects viewed this through prisms and mirrors, and the subjects' eyes were $41 \mathrm{~cm}$ from the display. Visible display resolution was $1,024 \times 538$ pixels.

We generated the stimuli in Matlab using the PsychToolbox ${ }^{48,49}$. A large diagonal cross of thin red lines crossed the entire display, aiding accurate fixation at the cross intersection. Stimuli were groups of circles randomly positioned at each presentation so all circles fell entirely within $0.75^{\circ}$ (radius) of fixation. To prevent perceptual grouping, individual circles were spread roughly homogeneously across this area (except in the high-density condition described below)

We used various stimulus configurations ${ }^{2}$ to ensure that low-level non-numerical stimulus features followed different time courses in different configurations. The first stimulus configuration ('constant area') kept summed circle surface areas constant across numerosities, ensuring equal luminance across numerosities. The second ('constant object size') kept individual circle size constant. The third ('constant perimeter') kept summed circle perimeters constant, ensuring equal edge extent across numerosities. The fourth ('high density') grouped the circles from the constant area figuration entirely within a $0.375^{\circ}$ radius area that was randomly placed inside the stimulus area.

Patterns were black circles on a grey background. Patterns were presented briefly $(300 \mathrm{~ms})$ to make sequentially counting impossible. They were refreshed with a new random pattern every $700 \mathrm{~ms}$, with $400 \mathrm{~ms}$ of grey background shown between pattern presentations. We presented each numerosity six times over 4,200 ms (2 repetition times, TRs), progressing slowly between numerosities. A proportion (10\%) of pattern presentations showed white circles instead of black. Subjects pressed a button when this happened, ensuring that they were paying attention to the patterns. Subjects responded to $80-100 \%$ of white circle presentations in each scanning run. No numerosity judgments were required.

The numerosities one to seven were first presented in ascending order, followed by 16.8 seconds showing 10 circles, followed seven to one in descending order, followed by 16.8 seconds with 20 circles. We acquired $44 \mathrm{fMRI}$ volumes during this cycle sequence, over 92.4 seconds, repeating this four times in each functional scanning run.

The long period of 20 circles allowed us to distinguish between very small and very large tuning widths $s^{2,50}$. This is far outside the response range of neurons with small preferred numerosities, producing little neural response. Conversely, neurons responding to stimulus contrast energy should respond most strongly to numerous circles.

As in many fMRI experiments, these stimuli probably cause some adaptation to repeated presentation of the same numerosities ${ }^{34,51}$. To minimize effects of adaptation on estimate numerosity preferences, we used a single model to summarize responses to both increasing and decreasing numerosity. This counterbalanced adaptation effects with stimuli that give both higher and lower responses preceding presentation of any numerosity, so reducing dependence on preceding stimuli.

MRI acquisition and preprocessing. We acquired MRI data on a 7T Philips Achieva scanner. Acquisition and pre-processing protocols are described fully in our previous study ${ }^{13}$. Briefly, we acquired T1-weighted anatomical scans, automatically segmented these with Freesurfer, then manually edited labels to minimize segmentation errors using ITK-SNAP. This provided a highly accurate cortical surface model at the grey-white matter border to characterize cortical organization. We acquired T2*-weighted functional images using a 32-channel head coil at a resolution of $1.77 \times 1.77 \times 1.75 \mathrm{~mm}$, with 41 interleaved slices of $128 \times 128$ voxels. The resulting field of view was $227 \times 227 \times 72 \mathrm{~mm}$. TR was $2,100 \mathrm{~ms}$, echo time (TE) was $25 \mathrm{~ms}$, and flip angle was $70^{\circ}$. We used a single-shot gradient echo sequence with SENSE acceleration factor 3.0 and anterior-posterior encoding. Maximum gradient strength was $26 \mathrm{mT} \mathrm{m}^{-1}$ and maximum slew rate was $140 \mathrm{~T} \mathrm{~m}^{-1} \mathrm{~s}^{-1}$. We used a third-order image-based $\mathrm{B} 0$ shim of the functional scan's field of view (in-house IDL software, v6.3, RSI, Boulder, CO).

This covered most of the brain, but omitted anterior frontal and temporal lobes, where 7T fMRI has low response amplitudes and large spatial distortions. Functional runs were each 176 time frames (369.6 seconds) in duration. The interval between runs was around 1 minute, the minimum possible. For each stimulus configuration, we acquired eight repeated runs in one session, with different configurations on different days.

We applied no spatial or temporal smoothing. We measured and corrected for head motion artefacts between and within functional scans. We then averaged functional data across each session's runs, aligned it to anatomical scans and interpolated it into each subject's anatomical segmentation space. We analysed responses from each configuration (session) separately and also averaged together.

fMRI data analysis. We estimated numerosity response models from fMRI data and stimulus time courses as previously described ${ }^{2,13}$, following a pRF modelling approach ${ }^{50}$. pRF models describe the aggregate tuning of neural populations within each grey matter fMRI recording site $(n=159,136$ recording sites across all subjects). Briefly, for each recording site and stimulus configuration, we used forward modelling to predict neural responses to the time course of displayed numerosities at each time point, for a set of candidate neural response models. We averaged all scanned stimulus cycles together, so each numerosity response model captured $44 \mathrm{fMRI}$ response measurements (that is, $n=44$ ). Each candidate neural response model described numerosity tuning using logarithmic Gaussian functions ${ }^{2,6,13}$ characterized by: (1) a preferred numerosity (mean of the Gaussian distribution) and (2) a tuning width (standard deviation of the Gaussian). A candidate time course for each candidate neural response model was calculated as the overlap of the stimulus at each time point with this response model. We convolved these with a haemodynamic response function (HRF) to generate candidate fMRI time courses. For each fMRI recording point, we chose the tuning parameters whose associated fMRI time course most closely fitted the recorded data, by minimizing the sum of squared errors (maximizing $R^{2}$, variance explained) between the predicted and observed fMRI time series. Because HRF parameters differ between subjects, but differ little between brain areas or recording sessions ${ }^{52}$, we then estimated subject-specific HRF parameters across the whole acquired fMRI volume from all the data recorded from each subject, as described elsewhere ${ }^{2,23}$, and re-fitted response models using these HRF parameters.

We demonstrate that logarithmic Gaussian functions explain more response variance than linear Gaussian functions by also fitting pRF models in which candidate neural response models were linear Gaussians ${ }^{2,6,13}$. We then compared the variance explained in all recording sites in each region of interest, ROI (grouped across all subjects) using a Wilcoxon signed-rank test.

To convert these variance-explained measures to probabilities of observing these model fits by chance, we using the same procedure to fit tuning models to recordings from 243,000 white matter recording points in the same scans, creating a null distribution ${ }^{13}$. We then determined the proportion of fits exceeding any variance explained. We used FDR correction for multiple comparison ${ }^{53}$, taking all grey matter recording sites in all subjects' scanning volumes into account.

We excluded from further analysis those recording sites for which pRF models explained less than $30 \%$ of response variance (that is, those with a probability above 0.0371 of observing this goodness of fit by chance, FDR-corrected).

Candidate preferred numerosities extended beyond the presented numerosity range, allowing model fit parameters beyond this range. This meant that returned parameters within the stimulus range were reported accurately, not just the best fit of a limited set. We could not accurately estimate preferred numerosities outside the stimulus range, so excluded any recording sites with preferred numerosities outside this range from further analysis.

Definitions of regions of interest. Here, we aimed to search for new numerosity maps throughout the cortical surface. However, our approach followed that in our previous demonstration of numerosity maps, which focused on the posterior parietal and dorsal occipital lobes ${ }^{2}$. Again, we started with the numerosity-selective 
response model from the average of all stimulus configurations. We rendered the variance explained of all recording points with preferred numerosities in the presented range onto the cortical surface (Supplementary Fig. 1). This highlighted six regions where numerosity-selective response models consistently captured responses well. These regions were located similarly (with respect to major sulci) in all hemispheres and formed the basis of our ROIs. We then rendered the preferred numerosities of each recording site on the cortical surface around these regions (Supplementary Fig. 2). Across hemispheres and stimulus configurations, we consistently found topographic representations of preferred numerosity at these locations $(6$ maps $\times 2$ hemispheres $\times 5$ subjects $=60$ maps, each measured repeatedly in four stimulus configurations on different days). In each map, we defined lines joining locations with equal preferred numerosity at the low and high ends of the preferred numerosity range seen in each numerosity map (the 'ends' of the map). The other map borders (the 'sides') followed the edges of topographic organization, where the goodness of model fits decreased.

Conversion to MNI coordinates. Our analyses were in individual subject space. To describe map locations on an average brain, we converted these to MNI $x, y$ and $z$ coordinates. We first located each individual subject's map centres on the cortical surface. We then transformed each subject's anatomical MRI data, together with these map centre locations, into MNI average template space with MINC's 'mincresample' tool (http://packages.bic.mni.mcgill.ca) using rigid alignment and linear scaling. We took the mean and standard deviation of the resulting MNI coordinates of each map across subjects.

Analysis of changes across the ROI. We calculated the distance along the cortical surface from each point in each ROI to the nearest point on the lines of the lowest and highest preferred numerosities. The ratio between the distances to each line gave a normalized distance along the ROI in the primary direction of change of numerosity preferences. We multiplied this by the mean ROI length in this direction.

We binned the recording points within every $2 \mathrm{~mm}$ along each numerosity map, calculating the mean and standard error of their preferred numerosities in each stimulus configuration and their average. Bins were excluded if their cortical surface extent was smaller than one fMRI voxel or smaller than the point-spread function of $7 \mathrm{~T} \mathrm{fMRI}^{54}$. Bin counts varied with the maps' cortical extents, ranging from 6 to 18, and are given in each figure. We fitted the best-fitting logarithmic functions to bootstrapped samples of the bin means, because preferred numerosity progressions are fitted better by logarithmic functions than straight lines ${ }^{2,13}$. From these bootstrapped fits, we took the median slope and intercept as the best-fitting numerosity progressions. We determined $95 \%$ confidence intervals by plotting all bootstrapped fit lines and finding the 2.5 and 97.5 percentiles of their values.

We determined the statistical significance of these slopes with a permutation analysis. We repeatedly (10,000 times) randomized which preferred numerosity was associated with each distance bin and fitted the slopes of each permutation. We then determined how many of these permutations had equal or greater slopes than the observed data, the probability of observing this slope by chance. We FDR-corrected this probability, taking into account probabilities from all stimulus configurations, maps, hemispheres and subjects.

Similarly, for each ROI we looked for changes in tuning width with preferred numerosity ${ }^{2,6,13}$. We binned the recording sites within every 0.25 increase in preferred numerosity, calculating the mean and standard error of their tuning width. Bins were excluded if their cortical surface extent was smaller than one fMRI voxel or smaller than the point-spread function of $7 \mathrm{~T} \mathrm{fMRI}{ }^{54}$. Few recording sites had preferred numerosities above 5, so we only used bins from 1 to 5 preferred numerosity. This formed a maximum of 17 preferred numerosity bins for each numerosity map; the number used in each map is given in each figure. We fitted linear functions to bootstrapped samples of bin means and took the median slope and intercept as the best-fitting tuning width progression. We used the permutation analysis described above to calculate the probability of observing this tuning width increase by chance.

Analysis of differences between stimulus configurations and numerosity maps. We consistently found preferred numerosity progressions across the cortical surface in all stimulus configurations. However, numerosity preferences may vary between stimulus configurations, for example owing to object-size selectivity in the same recording sites ${ }^{2,13}$. We grouped recording sites from the same numerosity map and hemisphere across all subjects. Recording sites counts differed between maps and hemispheres, and are given on Supplementary Fig. 6. In each group of recording sites, we correlated the numerosity preferences measured in each stimulus configuration with each other configuration using Pearson's correlation.

We tested whether the rate of preferred numerosity progression across the cortical surface was affected by stimulus configuration, numerosity map and hemisphere. For each subject, stimulus configuration, numerosity map and hemisphere, we took the slope of the best fitting logarithmic function to bins of preferred numerosity versus normalized cortical distance. We normalize cortical distance measures by total map length to avoid effects of map size differences between subjects and numerosity maps. We assessed differences between stimulus configurations, numerosity maps and hemispheres using a three-way ANOVA on these slopes $(n=240)$. We revealed where these differences reached significance by using subsequent multiple comparison tests ${ }^{21,22}$

We then tested whether the distribution of preferred numerosities within each numerosity map was affected by hemispheric lateralization, numerosity map identity and subject identity. For each numerosity map in each subject, we took each recording site's preferred numerosity from responses averaged across all stimulus configurations. To summarize the range of numerosity preferences with a single number, we then determined the upper quartiles of preferred numerosities present in each numerosity map in each subject and hemisphere. We previously assessed interquartile range differences, but lower quartiles did not differ significantly between hemispheres or numerosity maps, so interquartile range differences primarily reflected upper quartile differences.

We then assessed differences in the distribution of preferred numerosities across hemispheres, subjects and maps using a three-way ANOVA on the upper quartiles of all maps $(n=60)$. We revealed where these differences between hemispheres and maps reached significance by using subsequent multiple comparison tests.

We similarly assessed differences between the surface areas of the numerosity maps by determining the area of each numerosity map on the folded cortical surface. Again, we assessed differences in the distribution of preferred numerosities across hemispheres, subjects and maps using a three-way ANOVA on these surface areas, and we used subsequent multiple comparison tests to reveal which numerosity maps differed significantly in surface area.

Finally, we assessed tuning width differences between numerosity maps. Numerosity tuning widths vary systematically with preferred numerosity, and preferred numerosity distributions differ between maps. To examine differences in average tuning width without biases arising from different preferred numerosity distributions, we determined the value of the fitted tuning width progression for a preferred numerosity of three, an intermediate preferred numerosity that was present in almost all numerosity maps ${ }^{23}$. Again, we assessed tuning width differences across hemispheres, subjects and maps using a three-way ANOVA. Again, we used subsequent multiple comparison tests to reveal which numerosity maps differ significantly in tuning width.

Visual field mapping. We acquired visual field mapping responses to examine the relationship between numerosity maps and visual field maps. The visual field mapping paradigm was almost identical to that described in previous studies ${ }^{13,23,50,55}$. The stimulus consisted of drifting bar apertures at various orientations, which exposed a moving chequerboard pattern. The stimulus had a radius of $6.35^{\circ}$, larger than the numerosity mapping stimuli ( 0.75 radius). Two diagonal red lines, intersecting at the centre of the display, were again presented throughout the entire scanning run. Subjects pressed a button when these lines changed colour, and responded on $80-100 \%$ of colour changes within each scanning run.

Visual field mapping data were analysed following a standard $\mathrm{pRF}$ analysis, as described elsewhere ${ }^{23,50}$. We identified visual field map borders based on reversals in polar angle of visual field position preference and identified particular visual field maps with reference to previous studies ${ }^{24,56-58}$

Code availability. We performed fMRI analysis using mrVista, which is freely available at (http://white.stanford.edu/software/). Custom code is added to this repository on publication.

Data availability. The data that support the findings of this study are available from the corresponding author upon request.

\section{Received 24 June 2016; accepted 9 December 2016; published 23 January 2017}

\section{References}

1. Wandell, B. A., Dumoulin, S. O. \& Brewer, A. A. Visual field maps in human cortex. Neuron 56, 366-383 (2007).

2. Harvey, B. M., Klein, B. P., Petridou, N. \& Dumoulin, S. O. Topographic representation of numerosity in the human parietal cortex. Science 341, 1123-1126 (2013).

3. Vallentin, D. \& Nieder, A. Behavioral and prefrontal representation of spatial proportions in the monkey. Curr. Biol. 18, 1420-1425 (2008).

4. Piazza, M. Neurocognitive start-up tools for symbolic number representations Trends Cogn. Sci. 14, 542-551 (2010)

5. Knops, A., Piazza, M., Sengupta, R., Eger, E. \& Melcher, D. A shared, flexible neural map architecture reflects capacity limits in both visual short-term memory and enumeration. J. Neurosci. 34, 9857-9866 (2014).

6. Nieder, A. \& Miller, E. K. Coding of cognitive magnitude: compressed scaling of numerical information in the primate prefrontal cortex. Neuron 37, 149-157 (2003)

7. Dehaene, S. The Number Sense: How the Mind Creates Mathematics (Oxford Univ. Press, 1997).

8. Halberda, J., Mazzocco, M. M. \& Feigenson, L. Individual differences in non-verbal number acuity correlate with maths achievement. Nature 455, 665-668 (2008). 
9. Harvey, B. M. Quantity cognition: numbers, numerosity, zero and mathematics. Curr. Biol. 26, R419-R421 (2016).

10. Chen, B. L., Hall, D. H. \& Chklovskii, D. B. Wiring optimization can relate neuronal structure and function. Proc. Natl Acad. Sci. USA 103, 4723-4728 (2006).

11. Durbin, R. \& Mitchison, G. A dimension reduction framework for understanding cortical maps. Nature 343, 644-647 (1990).

12. Ramón y Cajal, S. Textura del Sistema Nervioso del Hombre y de los Vertebrados (Madrid Nicolas Moya, 1904).

13. Harvey, B. M., Fracasso, A., Petridou, N. \& Dumoulin, S. O. Topographic representations of object size and relationships with numerosity reveal generalized quantity processing in human parietal cortex. Proc. Natl Acad. Sci. USA 112, 13525-13530 (2015).

14. Eger, E. et al. Deciphering cortical number coding from human brain activity patterns. Curr. Biol. 19, 1608-1615 (2009)

15. Eger, E., Pinel, P., Dehaene, S. \& Kleinschmidt, A. Spatially invariant coding of numerical information in functionally defined subregions of human parietal cortex. Cereb. Cortex 25, 1319-1329 (2015).

16. Nieder, A., Freedman, D. J. \& Miller, E. K. Representation of the quantity of visual items in the primate prefrontal cortex. Science 297, 1708-1711 (2002).

17. Nieder, A. \& Miller, E. K. A parieto-frontal network for visual numerical information in the monkey. Proc. Natl Acad. Sci. USA 101, 7457-7462 (2004).

18. Piazza, M., Izard, V., Pinel, P., Le Bihan, D. \& Dehaene, S. Tuning curves for approximate numerosity in the human intraparietal sulcus. Neuron $\mathbf{4 4}$, 547-555 (2004).

19. Piazza, M., Pinel, P., Le Bihan, D. \& Dehaene, S. A magnitude code common to numerosities and number symbols in human intraparietal cortex. Neuron 53, 293-305 (2007).

20. Wandell, B. A., Brewer, A. A. \& Dougherty, R. F. Visual field map clusters in human cortex. Phil. Trans. R. Soc. Lond. B 360, 693-707 (2005).

21. Hochberg, Y. \& Tamhane, A. C. Multiple Comparison Procedures (Wiley, 1987).

22. Searle, S. R., Speed, F. M. \& Milliken, G. A. Population marginal means in the linear model: an alternative to least squares means. Am. Stat. 34, 216-221 (1980).

23. Harvey, B. M. \& Dumoulin, S. O. The relationship between cortical magnification factor and population receptive field size in human visual cortex: constancies in cortical architecture. J. Neurosci. 31, 13604-13612 (2011).

24. Hagler, D. J. Jr \& Sereno, M. I. Spatial maps in frontal and prefrontal cortex. Neuroimage 29, 567-577 (2006).

25. Bueti, D. \& Walsh, V. The parietal cortex and the representation of time space, number and other magnitudes. Phil. Trans. R. Soc. Lond. B 364, 1831-1840 (2009).

26. Hayashi, M. J. et al. Interaction of numerosity and time in prefrontal and parietal cortex. J. Neurosci. 33, 883-893 (2013).

27. Dehaene, S., Izard, V., Spelke, E. \& Pica, P. Log or linear? Distinct intuitions of the number scale in western and Amazonian indigene cultures. Science 320, 1217-1220 (2008).

28. Walsh, V. A theory of magnitude: common cortical metrics of time, space and quantity. Trends Cogn. Sci. 7, 483-488 (2003).

29. Santens, S. \& Gevers, W. The SNARC effect does not imply a mental number line. Cognition 108, 263-270 (2008).

30. van Dijck, J. P. \& Fias, W. A working memory account for spatial-numerical associations. Cognition 119, 114-119 (2011).

31. Dehaene, S., Bossini, S. \& Giraux, P. The mental representation of parity and number magnitude. J. Exp. Psychol. Gen. 122, 371-396 (1993).

32. Anobile, G., Cicchini, G. M. \& Burr, D. C. Number as a primary perceptual attribute: a review. Perception 45, 5-31 (2016).

33. Hyde, D. C. Two systems of non-symbolic numerical cognition. Front. Hum. Neurosci. 5, 150 (2011).

34. Piazza, M., Fumarola, A., Chinello, A. \& Melcher, D. Subitizing reflects visuo-spatial object individuation capacity. Cognition 121, 147-153 (2011).

35. Dehaene, S., Piazza, M., Pinel, P. \& Cohen, L. Three parietal circuits for number processing. Cogn. Neuropsychol. 20, 487-506 (2003)

36. Dehaene, S., Spelke, E., Pinel, P., Stanescu, R. \& Tsivkin, S. Sources of mathematical thinking: behavioral and brain-imaging evidence. Science 284, 970-974 (1999).

37. Domenech, P. \& Koechlin, E. Executive control and decision-making in the prefrontal cortex. Curr. Opin. Behav. Sci. 1, 101-106 (2015).

38. Kiani, R. \& Shadlen, M. N. Representation of confidence associated with a decision by neurons in the parietal cortex. Science 324, 759-764 (2009).

39. Cohen Kadosh, R. et al. Virtual dyscalculia induced by parietal-lobe TMS impairs automatic magnitude processing. Curr. Biol. 17, 689-693 (2007).
40. Viswanathan, P. \& Nieder, A. Differential impact of behavioral relevance on quantity coding in primate frontal and parietal neurons. Curr. Biol. 25, 1259-1269 (2015).

41. Ramirez-Cardenas, A., Moskaleva, M. \& Nieder, A. Neuronal representation of numerosity zero in the primate parieto-frontal number network. Curr. Biol. 26, 1285-1294 (2016).

42. Arcaro, M. J., Pinsk, M. A., Li, X. \& Kastner, S. Visuotopic organization of macaque posterior parietal cortex: a functional magnetic resonance imaging study. J. Neurosci. 31, 2064-2078 (2011).

43. Kolster, H. et al. Visual field map clusters in macaque extrastriate visual cortex J. Neurosci. 29, 7031-7039 (2009).

44. Orban, G. A., Van Essen, D. \& Vanduffel, W. Comparative mapping of higher visual areas in monkeys and humans. Trends Cogn. Sci. 8, 315-324 (2004).

45. Shuman, M. \& Kanwisher, N. Numerical magnitude in the human parietal lobe; tests of representational generality and domain specificity. Neuron 44, 557-569 (2004).

46. Gobel, S. M., Johansen-Berg, H., Behrens, T. \& Rushworth, M. F. Response-selection-related parietal activation during number comparison. J. Cogn. Neurosci. 16, 1536-1551 (2004).

47. Cavdaroglu, S., Katz, C. \& Knops, A. Dissociating estimation from comparison and response eliminates parietal involvement in sequential numerosity perception. Neuroimage 116, 135-148 (2015).

48. Brainard, D. H. The psychophysics toolbox. Spat. Vis. 10, 433-436 (1997)

49. Pelli, D. G. The VideoToolbox software for visual psychophysics: transforming numbers into movies. Spat. Vis. 10, 437-442 (1997).

50. Dumoulin, S. O. \& Wandell, B. A. Population receptive field estimates in human visual cortex. Neuroimage 39, 647-660 (2008).

51. Burr, D. \& Ross, J. A visual sense of number. Curr. Biol. 18, 425-428 (2008).

52. Handwerker, D. A., Ollinger, J. M. \& D'Esposito, M. Variation of BOLD hemodynamic responses across subjects and brain regions and their effects on statistical analyses. Neuroimage 21, 1639-1651 (2004).

53. Chumbley, J., Worsley, K., Flandin, G. \& Friston, K. Topological FDR for neuroimaging. Neuroimage 49, 3057-3064 (2010)

54. Shmuel, A., Yacoub, E., Chaimow, D., Logothetis, N. K. \& Ugurbil, K. Spatio-temporal point-spread function of fMRI signal in human gray matter at 7 tesla. Neuroimage 35, 539-552 (2007).

55. Zuiderbaan, W., Harvey, B. M. \& Dumoulin, S. O. Modeling center-surround configurations in population receptive fields using fMRI. J. Vis. 12, 10 (2012)

56. Amano, K., Wandell, B. A. \& Dumoulin, S. O. Visual field maps, population receptive field sizes, and visual field coverage in the human $\mathrm{MT}+$ complex. J. Neurophysiol. 102, 2704-2718 (2009).

57. Larsson, J. \& Heeger, D. J. Two retinotopic visual areas in human lateral occipital cortex. J. Neurosci. 26, 13128-13142 (2006).

58. Swisher, J. D., Halko, M. A., Merabet, L. B., McMains, S. A. \& Somers, D. C. Visual topography of human intraparietal sulcus. J. Neurosci. 27, 5326-5337 (2007).

\section{Acknowledgements}

This work was supported by Netherlands Organization for Scientific Research grants no. 452.08.008 to S.O.D. and no. 433.09.223 to S.O.D. and F. W. Cornelissen, and by Portuguese Foundation for Science and Technology grant no. IF/01405/2014 to B.M.H The Spinoza Centre is a joint initiative of the University of Amsterdam, Academic Medical Centre, VU University, VU Medical Centre, Netherlands Institute for Neuroscience and the Royal Netherlands Academy of Arts and Sciences. The funders had no role in study design, data collection and analysis, decision to publish or preparation of the manuscript.

\section{Author contributions}

B.M.H. and S.O.D. designed the study; B.M.H. collected and analysed data; B.M.H. wrote the manuscript with input from S.O.D.

\section{Additional information}

Supplementary information is available for this paper.

Reprints and permissions information is available at www.nature.com/reprints. Correspondence and requests for materials should be addressed to B.M.H.

How to cite this article: Harvey, B. M. \& Dumoulin, S. O. A network of topographic numerosity maps in human association cortex. Nat. Hum. Behav. 1, 0036 (2017)

\section{Competing interests}

The authors declare no competing interests. 